\title{
The Profile of Multi Drug Resistant Bacterial Infections in Long Term Care Facility
}

\section{EL Bedewy RMS}

Geriatrics and Gerontology Department, Faculty of Medicine- Ain Shams University

\begin{abstract}
Background: Data about infections in LTCFs is limited
\end{abstract}

Aim: To detect the commonest multidrug resistant bacteria and its antibiotic sensitivity in elderly residents at LTCF

Methods: 126 LTCF elderly residents and documented multi drug resistant organisms in their samples. Culture and sensitivity was done for collected samples documenting multidrug resistant organisms with its antibiotic susceptibility to a range of different antibiotic groups. Demographic data was collected and comorbidities were established. All samples were processed as per standard techniques and bacteria identified by standard biochemical tests. IBM SPSS statistics (version 24, IBM corp., USA, 2016) was used for data analysis.

Results: The age range from 60 to 98 years with mean $70.5794+/$ SD 10.17 years. Males are $79(\sim 62.27 \%)$, while females are $47(\sim 37.37 \%)$, The predominant resistant detected organism in these samples is Proteus $(49.2 \%)$, then Klebsiela $(22.2 \%)$, then E Coli $(11.1 \%)$, then Pseudomonas (9.5\%), then MRSA $(4.8 \%)$ and lastly, Mix of [Klebsiela + Pseudomonas ] (3.2\%). Proteus is found to be highly statistically significant sensitive to Meronam (MEM), Imipenam (IMP), Ciprofloxacin (CP) and highly statistically significant resistant to Cefuroxime (CRM), Ceftazidime (CAZ), Gentamycin (GM), Colistin (CT), Trimethoprim/Sulfamethoxazole (TS), Nitrofurantoin (FD), Tobramycin (Tobra) while Klebsiella is highly statistically significant sensitive to Ceftazidime (CAZ), Colistin (CT) and highly statistically significant resistant to Imipenam (IMP) and E Coli is found to be highly statistically significant sensitive to Gentamycin (GM) and Nitrofurantoin (FD) while Pseudomonas is highly statistically significant sensitive to Tobramycin and highly statistically significant resistant to CD,MEM, Minocyclin (MN), Vancomycin .MRSA is found to be highly statistically significant sensitive to to Trimethoprim/Sulfamethoxazole (TS), Clindamycin (CD), Minocyclin (MN), and Vancomycin while the mix of Klebsiella + Pseudomonas is highly statistically significant sensitive to Tobramycin.

Conclusions: Gram negative bacteria are the most common multidrug resistant bacteria at long term care facility mostly Proteus, the best antibiotics to use for proteus infections was Minocyclin (MN) then Meronam (MEM) then Imipenam (IMP).

Keywards: Antibiotic susceptibility, multi drug resistant bacteria, long term care facility, elderly residents.

\section{Background}

As the population is aging so the functional dependency is increasing in the society and the need for long - term care facilities (LTCFs) is increasing. Residence at a Long term care facility (LTCF) and older age are both recognized as significant risk factors for harboring multidrug resistant Gram-negative bacteria (MDRGNB)

1. Antimicrobial resistance problem is growing and need further synchronized confrontation weapons. The Centers for Disease Control and Prevention estimates that in the United States, >2 million people become infected with a multidrug-resistant organism (MDRO) each year; subsequently, at least 23,000 die from these infections and from complications resulting from these illnesses ${ }^{2}$. Data about infections in LTCFs are limited ${ }^{3}$. Yet, in 
long-term care, up to $75 \%$ of antimicrobial use is inappropriate or unnecessary. Thus, one of the most pressing areas in need for antimicrobial stewardship is in long-term care facilities. ${ }^{4}$.

The aim of this study was to detect the commonest multidrug resistant bacteria and its antibiotic sensitivity in long term care facility residents.

\section{Method:}

A descriptive study for 126 long term care facility elderly residents having multi drug resistant organisms in their biological samples (urine, sputum, purulent dischages from ear, eye, pressure sore, or percutanous endoscopic gastrostomy (PEG) tube site).

All samples were processed as per standard techniques and bacteria identified by standard biochemical tests. Demographic data collected and comorbidities established for included cases.

\section{Statistical method}

IBM SPSS statistics (version 24, IBM corp., USA, 2016) was used for data analysis. Data were expressed as Mean $\pm \mathrm{SD}$ for quantitative parametric measures in addition to both number and percentage for categorized data. Chi square test was used to study the association between each 2 variables or comparison between 2 independent groups as regards the categorized data. The probability of error at 0.05 was considered significant, while at 0.01 and 0.001 are highly significant.

\section{Results:}

A sample of 126 elderly long term care facility (LTCF) residents having multi drug resistant bacterial infections with age range from 60 to 98 years with mean $70.57+/$ SD 10.17years.

Males are $79(\sim 62.27 \%)$, while females are $47(\sim 37.37 \%)$, The resistant organism detected in these samples were Proteus (49.2 \%), Klebsiela (22.2\%), E Coli (11.1\%), Pseudomonas (9.5\%), MRSA (4.8\%) and lastly, Mix of [Klebsiella + Pseudomonas ] (3.2\%).

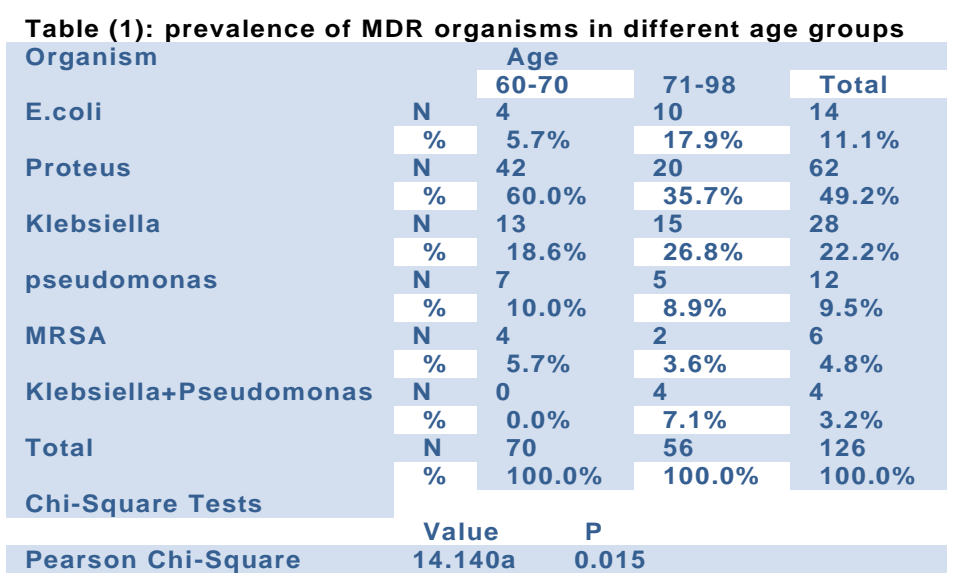

follow: $59.3 \%$ of urine, $50 \%$ of sputum samples, $53.8 \%$ of bed sores, $50 \%$ of tracheostomy site , $15.4 \%$ of PEG sites, $0 \%$ of ear discharge, finally $25 \%$ of eye discharge. Proteus is isolated mainly from samples obtained from patients with the comorbidity of Anoxic Brain Damage (ABD).

Table (2): gender difference of MDR bacterial infection profiles

\begin{tabular}{|c|c|c|c|c|}
\hline \multicolumn{2}{|l|}{ Organism } & $\begin{array}{l}\text { Sex } \\
\text { male }\end{array}$ & \multirow{2}{*}{$\begin{array}{l}\text { Female } \\
4\end{array}$} & \multirow[t]{2}{*}{ Total } \\
\hline E.coli & $\mathbf{N}$ & 10 & & \\
\hline & $\%$ & $12.7 \%$ & $8.5 \%$ & $11.1 \%$ \\
\hline Proteus & $\mathbf{N}$ & 45 & 17 & 62 \\
\hline & $\%$ & $57.0 \%$ & $36.2 \%$ & $49.2 \%$ \\
\hline Klebsiella & $\mathbf{N}$ & 14 & 14 & \\
\hline & $\%$ & $17.7 \%$ & $29.8 \%$ & $22.2 \%$ \\
\hline Pseudomonas & $\mathbf{N}$ & 6 & 6 & 12 \\
\hline & $\%$ & $7.6 \%$ & $12.8 \%$ & $9.5 \%$ \\
\hline MRSA & $\mathbf{N}$ & 2 & 4 & 6 \\
\hline & $\%$ & $2.5 \%$ & $8.5 \%$ & $4.8 \%$ \\
\hline Klebsiella+Pseudomonas & $\mathbf{N}$ & 2 & 2 & \\
\hline & $\%$ & $2.5 \%$ & $4.3 \%$ & $3.2 \%$ \\
\hline Total & $\mathbf{N}$ & 79 & 47 & 126 \\
\hline & $\%$ & $100.0 \%$ & $100.0 \%$ & $100.0 \%$ \\
\hline Chi-Square Tests & & & & \\
\hline Pearson Chi-Square & $\begin{array}{l}\text { Value } \\
8.291 \mathrm{a}\end{array}$ & $\begin{array}{l}P \\
.141\end{array}$ & & \\
\hline
\end{tabular}

\section{The Antibiotics efficiency for different MDR bacteria isolated in this study is as the following:}

- Proteus responded best to Minocyclin, Meronam, and imepenem. It was highly resistant and nitrofurantoin

- Klebsiella responded best to Meronam, colistin and Amikacin. The antibiotic with highest resistance in klebsiella was Cefotaxime.

- E Coli was highly sensitive to Meronam, minocycline and Colistin of cases; Gentamycin was the most resistant Antibiotic in E Coli infections.

- Pseudomonas responded best to Colistin, it was highly resistant to Meronam and Vancomycin.

- Correlations of organisms with comorbidities:

- $42.9 \%$ of $\mathrm{E}$ Coli occurred in patients with parkinsonism and dementia, 33.3\% of Pseudomonas have Diabetes Mellitus (DM) + Cerebrovascular stroke $(\mathrm{CVS})+$ Dementia + Renal impairment $(\mathrm{RI})+$ Pressure ulacer (PU) + Post cardiac arrest(PCA), $28.6 \%$ of Klebsiella have CVS, $25.8 \%$ of Proteus have Anoxic Brain Damage (ABD). $50 \%$ of patients with the mix (Klebsiella +Pseudomonas) have DM $+\mathrm{RI}+$ Dementia and the other $50 \%$ have DM + Hypertension $(\mathrm{HTN})+\mathrm{CVS}+\mathrm{PU}$.

- $33.3 \%$ of patients with MRSA have ABD, 33.3\% of patients with MRSA have DM + CVS+ Dementia, and $33.3 \%$ have DM + PU+ Dementia. 
Table (3): Sites of MDR bacteria isolation among the participants

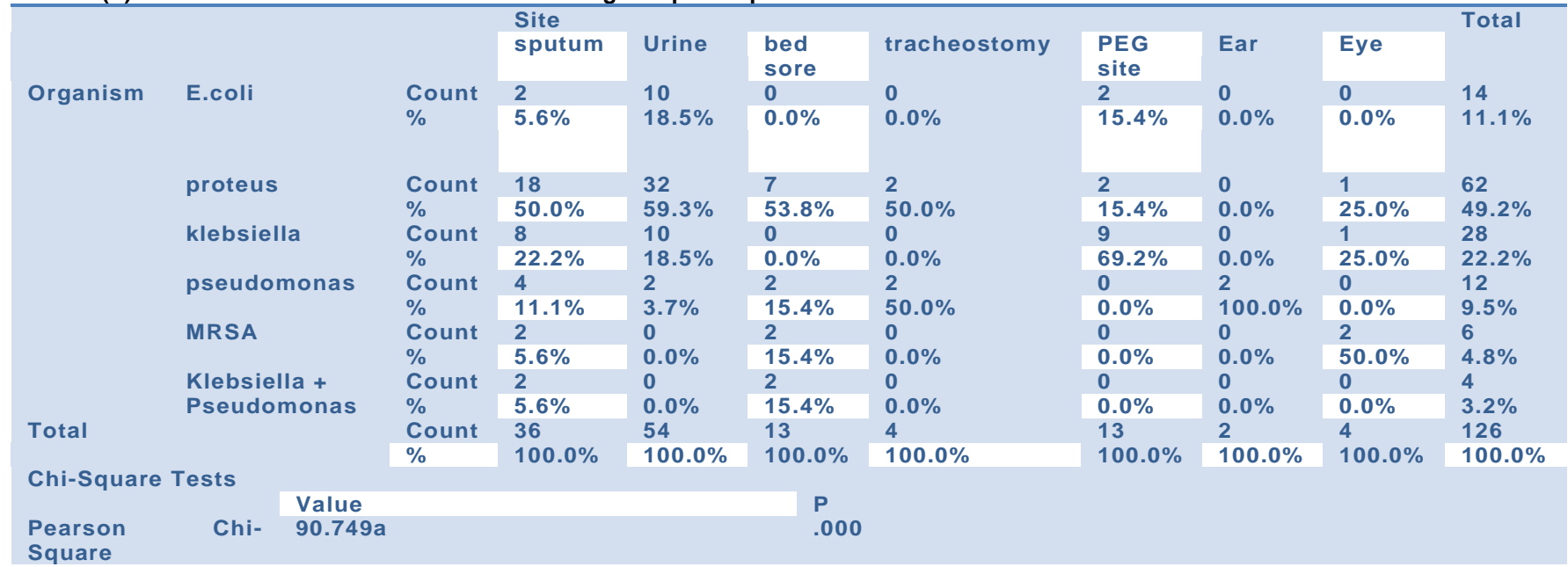

Square

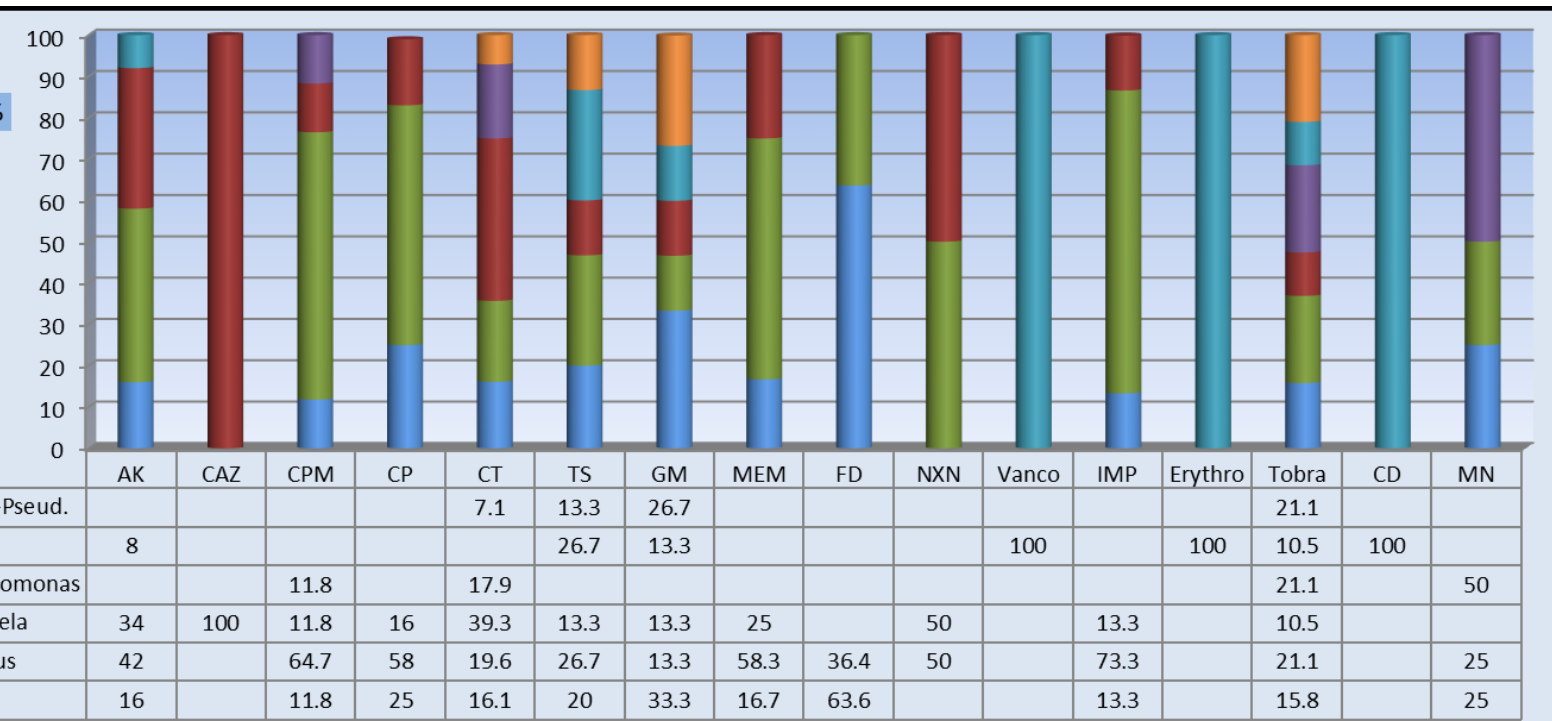

Figure (1): Frequency of all studied antibiotics as regards their sensitivities.

\{Amikin (AK), Ceftazidime (CAZ), Cefepime (CPM), Ciprofloxacin (CP), Colistin (CT), Trimethoprim/Sulfamethoxazole (TS),

Gentamycin (GM), Meronam (MEM), nitrofurantoin (FD), Norfloxacin (NXN), Vancomycin (Vanco), Imipenam (IMP), Erythromycin(Erythro), Tobramycin (Tobra), Clindamycin (CD), Minocyclin (MN)\}.

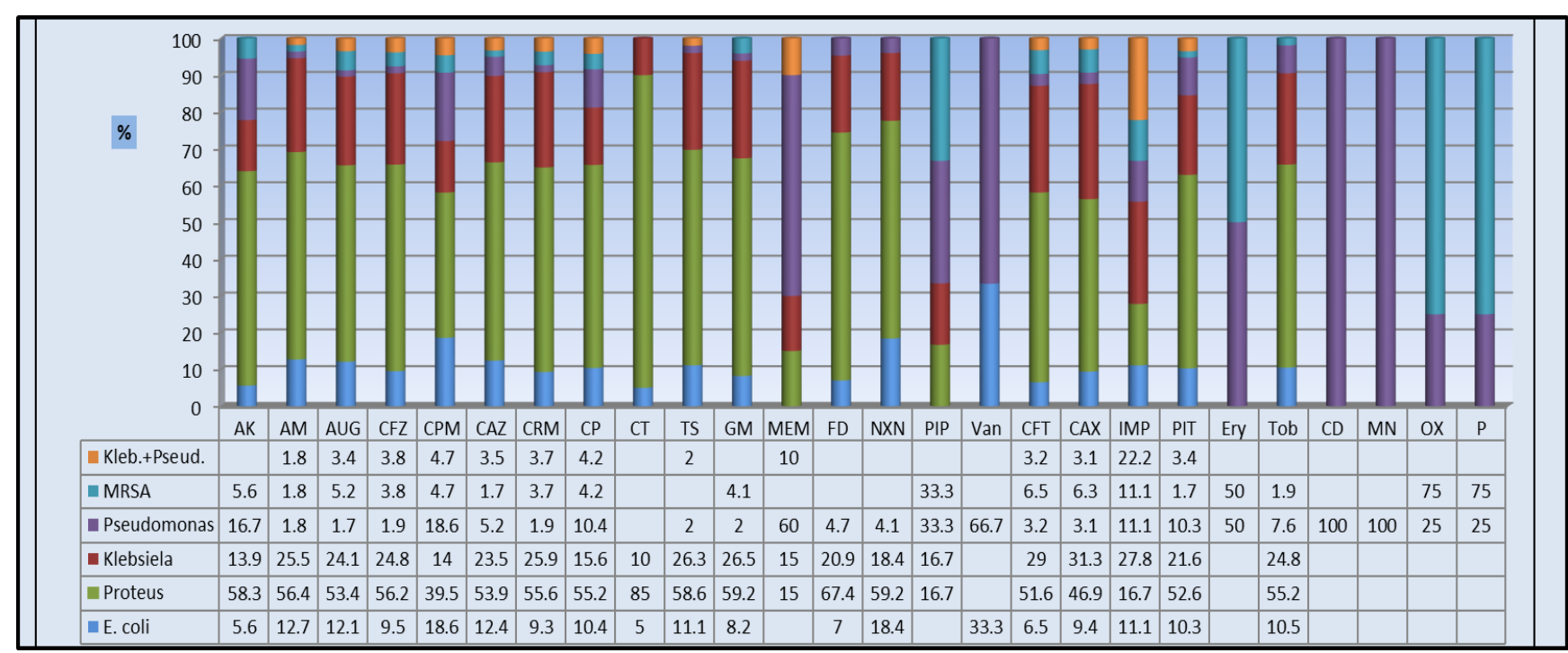

Fig. (2): Frequency of all studied antibiotics as regards their resistance.

\{ Amikin (AK), Ampicillin (AM), Augmentin (AUG), Cefazolin (CFZ), Cefepime (CPM), Ceftazidime (CAZ), Cefuroxime (CRM), Ciprofloxacin (CP), Colistin (CT), Trimethoprim/Sulfamethoxazole (TS), Gentamycin (GM), Meronam (MEM), Nitrofurantoin (FD). Norfloxacin (NXN), peperacillin (PIP). Vancomvcin( Vanco). Ceftriaxone (CFT). Cefotaxime (CAX). Imipenam (IMP). 
The best antibiotics sensitivity of each detected resistant organism under current study:

Figure(3) The best antibiotics sensitivity of E. coli (Above or equal $50 \%$ sensitivity) (The most preferable are MEM or $\mathrm{MN}$ )

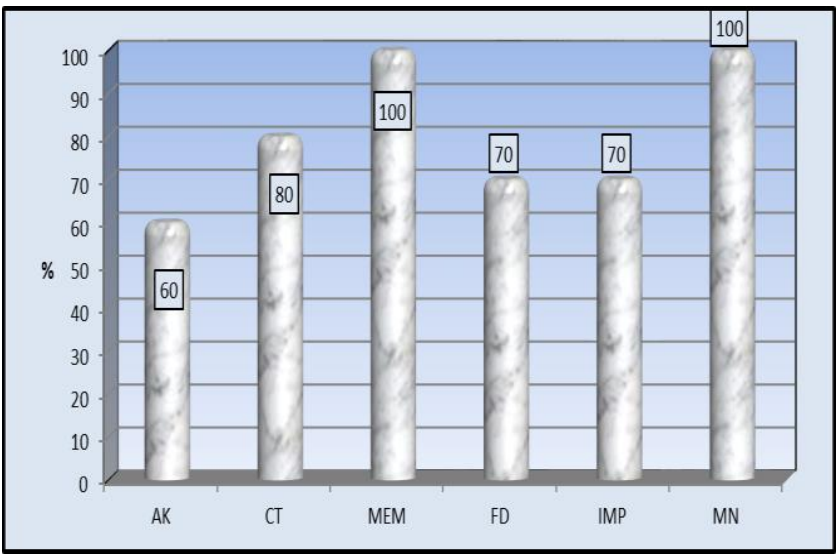

Figure (4): The best antibiotics sensitivity of Proteus (Above or equal $50 \%$ )

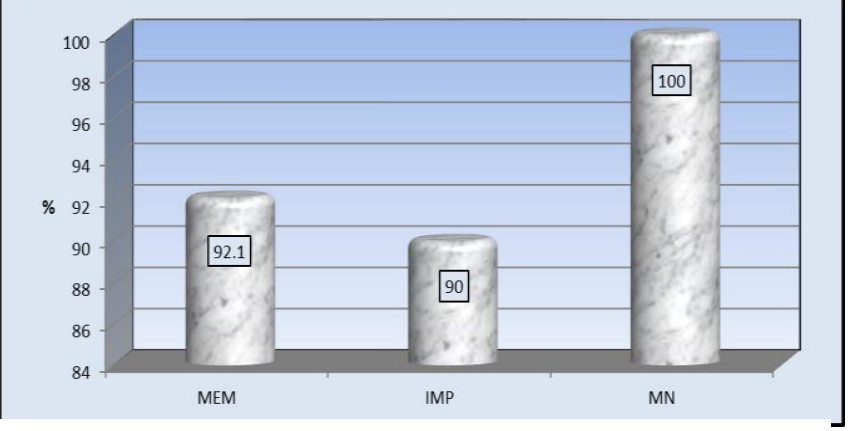

Figure (5): The best antibiotics sensitivity of Klebsiella (Above or equal $50 \%$ )

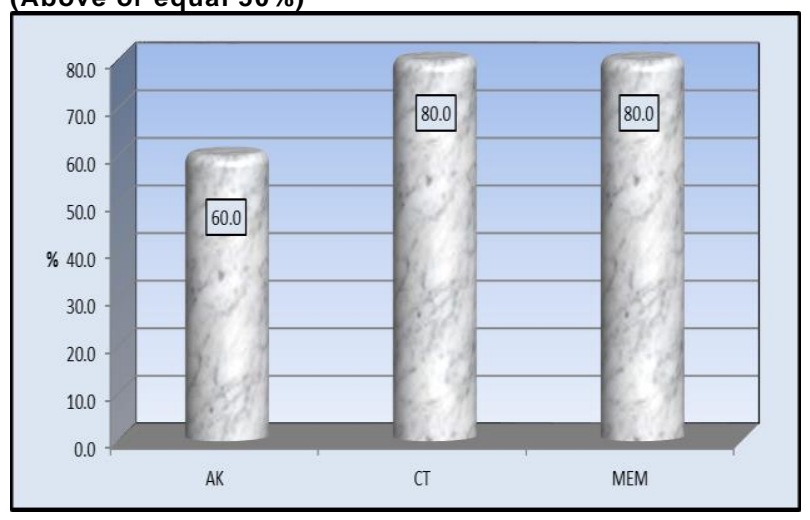

Figure (6): The best antibiotics sensitivity of Pseudomonas (Above or equal $50 \%$ )

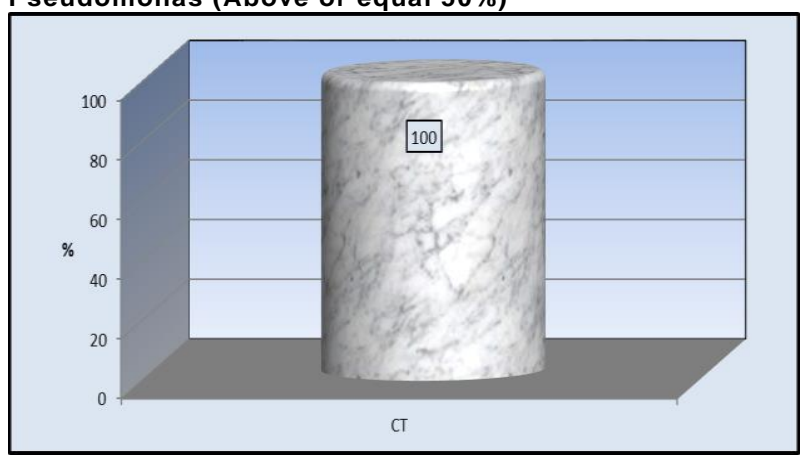

Figure (7): The best antibiotics sensitivity of MRSA (Above or equal $50 \%$ )

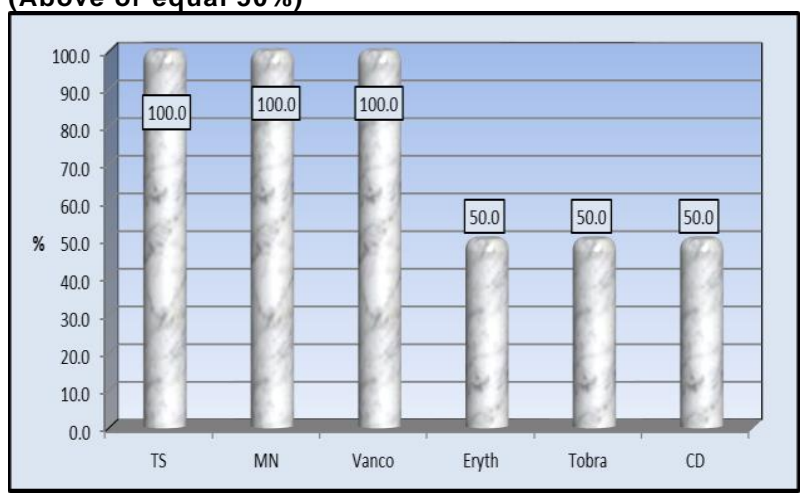

\section{Discussion:}

Infections with MDROs are associated with higher mortality rates, prolonged hospital length of stays, increased need for intensive care, and higher care costs. ${ }^{5}$ Moreover, older persons are also particularly susceptible to adverse effects of antimicrobials, including Clostridium difficile infections. ${ }^{6}$ thus, antibiotic stewardship is a mandatory health intervention among LTC residents.

In the current study, gram negative bacteria are the most common multidrug resistant bacteria at long term care facility mostly Proteus. Similarly, Pop-Vicas et al, found that the prevalence of MDR gram negative bacteria in elderly residents living in an LTCF was substantially higher than the prevalence of VRE and MRSA. ${ }^{7}$

In this study, Proteus best sensitivity was towards Minocyclin (MN) then Meronam (MEM) then Imipenam (IMP). Klebsiella best sensitivity was to Colistin (CT) and Meronam (MEM) then Amikin (AK) while Pseudomonas best sensitivity was to Colistin (CT). The resistance was high for ciprofloxacin, nitrofurantoin, and Trimethoprim/Sulfamethoxazole 
In previous results, a substantial proportion of MDR gram negative isolates recovered were resistant to commonly prescribed oral antimicrobials, with $85 \%$, $83 \%$, and $57 \%$ of isolates resistant to ciprofloxacin, firstgeneration cephalosporins, and trimethoprimsulfamethoxazole, respectively. ${ }^{7}$

\section{Conclusion:}

According to current study results, gram negative bacteria are the most common multidrug resistant bacteria at long term care facility mostly Proteus. Proteus best sensitivity is towards Minocyclin (MN) then Meronam (MEM) then Imipenam (IMP). So, these antibiotics clinically are preferable to be used with Proteus but if these antibiotics improperly are used against Proteus, it will lose these sensitivities and become resistant to them. The same can be concluded for other detected resistant organisms in current study as Klebsiella best sensitivity is to Colistin (CT) and Meronam (MEM) then Amikin (AK) while Pseudomonas best sensitivity is to Colistin (CT) on the other hand MRSA best sensitivity is towards Trimethoprim/Sulfamethoxazole (TS), Minocyclin $(\mathrm{MN})$, and Vancomycin then Erythromycin, Tobramycin and Clindamycin (CD). The mix of Klebsiella + Pseudomonas has best sensitivity to Colistin (CT), Gentamycin (GM), and Tobramycin then Trimethoprim/Sulfamethoxazole (TS). Those antibiotic sensitivities may be due to infrequent use or proper use of those antibiotics with corresponding organism till now.

\section{Recommendations:}

This study recommends the proper use of preferable antibiotics for each resistant organism at long term care facility and also recommends further studies at LTCF elderly to confirm its findings.

\section{References:}

1. 1- Choi JP, Cho EH, Lee SJ, Lee ST, Koo MS, Song YG. Influx of multidrug resistant Gram-negative bacteria (MDRGNB) in a public hospital among elderly patients from long-term care facilities: a singlecenter pilot study. Arch Gerontol Geriatr. 2012 Mar-Apr;54(2):e19-22. doi: 10.1016/j.archger.2011.05.026. Epub $2011 \mathrm{Jul} 20$.

2. 2- Centers for Disease Control and Prevention. Antibiotic resistance threats in the United States, 2013. 2014. Available from: http://www.cdc.gov/drugresistance/ threat-report-2013/ Accessed July 1, 2015.

3. 3- Centers for Disease Control and Prevention. Nursing Homes and Assisted Living (Long - term Care Facilities [LTCFs]). Available from: https://www.cdc.gov/longtermcare/index.html.

4. 4- Antimicrobial Stewardship in Long-Term Care Facilities: A Call to Action Review Article Journal of the American Medical Directors Association, Volume 17, Issue 2, 1 February 2016, Pages 183.e1183.e16

5. Mitchell SL, Shaffer ML, Loeb MB, et al. Infection Management and Multidrug-Resistant Organisms in Nursing Home Residents With Advanced Dementia. JAMA internal medicine. 2014;174(10):16601667. doi:10.1001/jamainternmed.2014.3918.

6. Kyne L, Merry C, O'Connell B, Kelly A, Keane C, O'Neill D. Factors associated with prolonged symptoms and severe disease due to. Clostridium difficile. Age Ageing. 1999;28(2):107-113.

7. Pop-Vicas, A., Mitchell, S. L., Kandel, R., Schreiber, R. and D'Agata, E. M. C. (2008), Multidrug-Resistant Gram-Negative Bacteria in a Long-Term Care Facility: Prevalence and Risk Factors. Journal of the American Geriatrics Society, 56: 1276-1280. doi:10.1111/j.15325415.2008.01787.x

Corresponding Author: Reem Mohamed Sabry EL Bedewy: drreem_sabry2005@yahoo.com 